\title{
MASS FLOWMETER USING A MULTI-SENSOR CHIP
}

\author{
Yong $\mathrm{Xu}$, Chen-Wei Chiu*, Fukang Jiang, Qiao Lin and Yu-Chong Tai \\ Electrical Engineering, California Institute of Technology, Pasadena, CA 91125, USA \\ "Umachines Inc., 3360 E. Foothill, Pasadena, CA 91107, USA
}

\begin{abstract}
We report here a novel mass flowmeter using a multisensor chip that includes a 1-D array of pressure, temperature and shear stress sensors. This shear stress sensor based flowmeter is capable of high sensitivity and wide measurement range. Our study also shows that the mass flowmeter using shear-stress sensors produces better resolution than that from pressure sensors in the laminar flow regime. Extensive tests have been carried out to evaluate the effects of overheat ratio, channel height and gas properties. We also find the $V^{2} \propto \tau^{1 / 3}$ law for conventional hot film sensors does not hold for our micromachined shear stress sensor.
\end{abstract}

\section{INTRODUCTION}

It is well known that pressure sensors can be used for mass flow rate measurements. Advantages of this approach includes negligible sensitivity to ambient temperature and a linear relationship between the pressure drop and flow rate in laminar flow regime. However, to obtain a high resolution, a significant pressure drop should be created which is not acceptable in some applications. Another scheme for mass flow rate measurements is based on heat transfer. A typical arrangement is a heater with two temperature sensors located upstream and downstream respectively [1]. For a given heating rate, the fluid mass flow rate is a function of the downstream-to-upstream temperature difference. The micromachined version of this structure has been presented by Lammerink et. al [2]. Other structures have also been explored, Ref. [3] gives a perfect review.

We previously developed a hot film shear stress sensor which shows excellent performance [4]. Since the shear stress is a function of velocity (or more appropriately, velocity gradient), if we install the sensor in a channel, we can measure the mass flow rate. Note that temperature sensor is needed for the temperature compensation of the hot film shear stress sensor. It would also be very helpful to know the pressure distribution of the channel flow. We fabricated a multisensor chip that includes a 1-D array of pressure, temperature and shear stress sensors. A novel mass flowmeter using this multi-sensor chip which shows high sensitivity and wide measurement range has been successfully developed.

\section{DESIGN, FABRICATION AND PACKAGING}

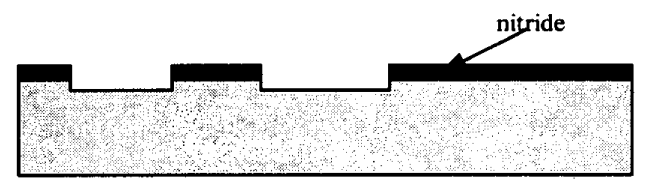

1. Deposit and pattern LPCVD nitride.

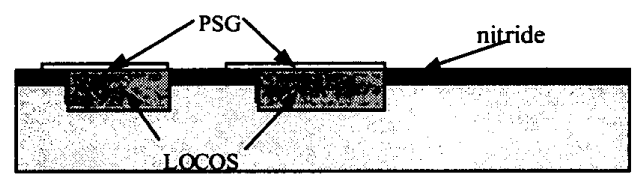

2. Local oxidation; deposit and pattern PSG.

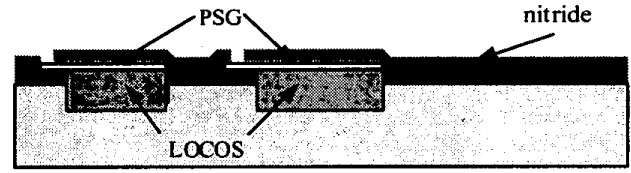

3. Deposit LPCVD nitride and open etch holes.

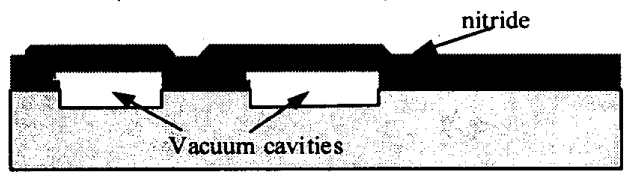

4. $49 \%$ HF etches PSG and oxide, and then seal the cavity by LPCVD nitride

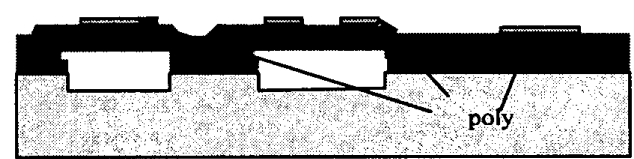

5. Deposit, dope, and pattern polysilicon.

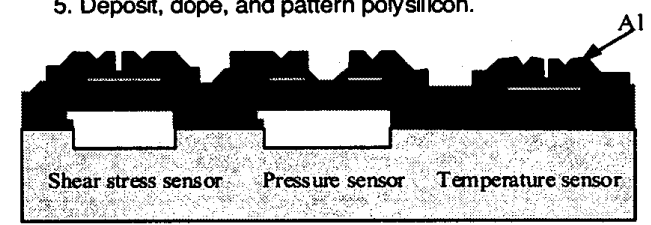

6. Deposit thin nitride, open contact holes, and then metallization.

Figure 1. Simplified fabrication process.

The multi-sensor chip contains five sensor clusters with a pitch of $2 \mathrm{~mm}$ consisting of shear stress, pressure and temperature sensors. The structure of the shear stress sensor is same as that in [4], and a simplified process can be seen in Figure 1. The sensing element, i.e. the thin film polysilicon resistor, is embedded in a $210 \times$ $210 \mu \mathrm{m}^{2}$ silicon nitride diaphragm with a vacuum cavity 
underneath to minimize the heat loss to the silicon substrate. The resistor is $7 \mu \mathrm{m}$ wide and $150 \mu \mathrm{m}$ long with a typical resistance of $1.4 \mathrm{k} \Omega$ at the room temperature. To increase the sensitivity of the pressure sensor, we implemented a bossed diaphragm design. The temperature sensor is a circular polysilicon thermistor with a typical resistance of $4 \mathrm{k} \Omega$. The process starts with the deposition and patterning of low stress silicon nitride on silicon wafers. Windows are etched further into silicon to form a $1 \mu \mathrm{m}$ trench. $2 \mu \mathrm{m}$ of thermal oxide is grown on the trench then the wafers are planarized by HF dip. A 4000 \& PSG (phosphosilicate glass) layer is deposited, patterned and annealed to form the etching channels. Next, about 1.5 $\mu \mathrm{m}$ low stress nitride is deposited and patterned, opening the etch holes. PSG and thermal oxide are etched away by $49 \% \mathrm{HF}$ and the nitride diaphragms are released. After this, the cavities are sealed by deposition of another layer of nitride and the diaphragms of the pressure sensors are patterned to form the beam and boss structures. $5000 \AA$ polysilicon

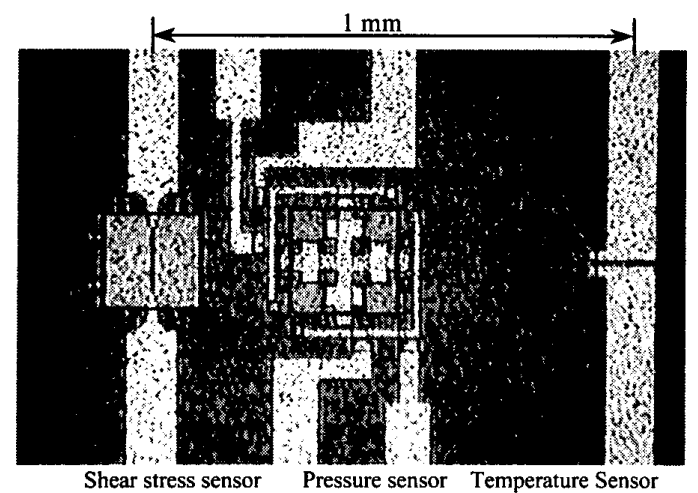

(a)

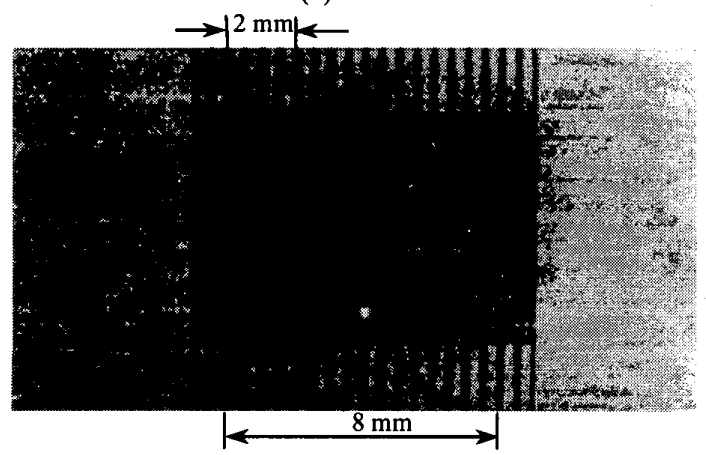

(b)

Figure 2 (a) Picture of one sensor cluster.

(b) Picture of a multi-sensor array chip.

films are deposited, doped and patterned to form the sensing resistors. The polysilicon films are actually doped twice. The first doping is a global boron ion implantation with a dose of $1 \times 10^{15} / \mathrm{cm}^{2}$ to obtain a nearly zero temperature coefficient of resistance (TCR). The second doping is a selective high dose boron ion implantation $\left(1 \times 10^{16} / \mathrm{cm}^{2}\right)$ on the shear stress, temperature sensors and also on the contacts and turning sections of the pressure resistors to minimize the non-effective resistance. Another $1800 \AA$ nitride is deposited as a passivation layer and is followed by contact holes opening and aluminum metallization. Finally the multi-sensor chip is diced into $2 \times 1 \mathrm{~cm}^{2}$ pieces and Figure 2 shows one sensor cluster and a complete multi-sensor chip.

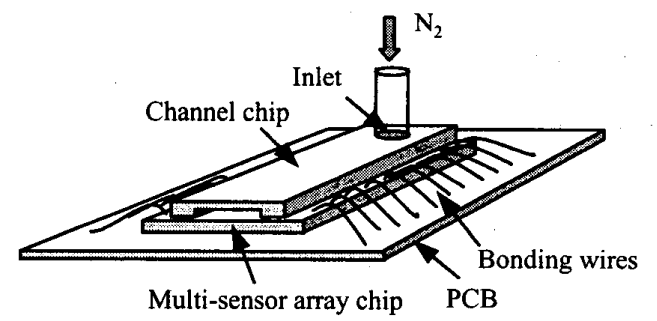

(a)

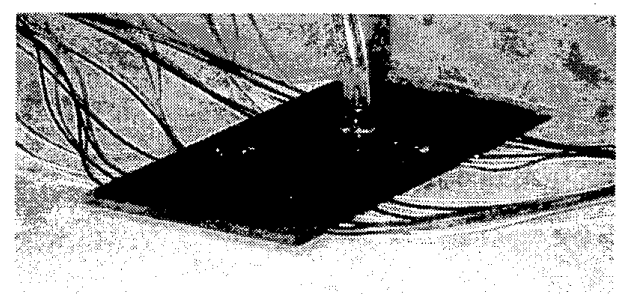

(b)

Figure 3 (a) Schematic of packaged device. (b) Real picture of packaged device.

To assemble the mass flowmeter, the multi-sensor chip is first bonded to a printed circuit board and then another channel chip ( $2.5 \mathrm{~mm}$ wide and $18 \mathrm{~mm}$ long) is flip bonded on top of the sensor chip as shown in Figure 3. To determine the effect of channel height, three different channels with heights of $150 \mu \mathrm{m}, 200 \mu \mathrm{m}$ and $288 \mu \mathrm{m}$ are fabricated by DRIE. The first sensor cluster is $4 \mathrm{~mm}$ away from the inlet to ensure all the sensors are in the fully developed flow regime. The pressure and temperature sensors are then calibrated before various flow measurements. Due to the bossed diaphragm design, the sensitivity of the pressure sensor is 570 $\mu \mathrm{V} / \mathrm{psi} \bullet \mathrm{V}$ which is much better than those using uniform diaphragms [5].

\section{OPERATING PRINCIPLES}

The operation of temperature and pressure sensors is very straightforward. Here we focus on the operation of shear stress sensor. Our shear stress sensor is a heated resistor sitting on a vacuum cavity. The heat loss of the resistor is a function of the velocity gradient, namely the wall shear stress of the ambient fluid defined by 


$$
\tau=\left.\mu \frac{d U}{d y}\right|_{y=0}
$$

where $\mu$ is the viscosity, $U$ is the streamwise velocity and $y$ is the direction normal to the sensor surface. The relationship between $\tau$ and the input power to the sensor is described by the $1 / 3$ law [1]:

$$
\frac{V^{2}}{R_{S}}=\Delta T\left(A(\rho \tau)^{1 / 3}+B\right)
$$

where $\Delta T$ is the temperature difference between the heated resistor and ambient, $A \propto C_{p}^{1 / 3} k_{T}^{2 / 3} / \mu^{1 / 3}\left(C_{p}\right.$ and $k_{T}$ are the heat capacity and thermal conductivity of the fluid respectively), $\rho$ is the density of the fluid and term $B$ represents the heat loss to the substrate. However equation (2) is derived for conventional macro heating element and may not be valid for our micromachined shear stress sensor. In this case, we should use the empirical formula

$$
\frac{V^{2}}{R_{S}}=\Delta T\left(A_{t}(\rho \tau)^{1 / n}+B_{t}\right)
$$

where $A_{t} B_{t}$ and $n$ must be determined empirically.

For the rectangular channel flow, if we assume the height is much smaller than the width (which is true in our case), the relationship between $\tau$ and the pressure gradient $d P / d x$ along the channel is [6]

$$
\tau=\frac{h}{2} \frac{d P}{d x} .
$$

where $h$ is the channel height. We already know that in laminar flow regime the flow rate $Q$ is function of $d P / d x[6]$

$$
Q=\frac{h^{3} w}{12 \mu} \frac{d P}{d x}
$$

So now we obtain a relationship between input power and the flow rate in laminar flow regime

$$
\frac{V^{2}}{R_{S}}=A_{0} Q^{1 / n}+B_{0}
$$

where $A_{0}=\Delta T A_{t}\left(\frac{6 \rho \mu}{h^{2} w}\right)^{1 / n}$ and $B_{0}=\Delta T B_{t}$.
The shear stress sensor works in constant temperature (CT) mode, namely the temperature of the sensor remains constant during operation. Figure 4 shows the simplified CT biasing circuits where $R_{S}$ is the shear stress sensor, $R_{l}$ is adjustable and $R_{2}$ equals $R_{3}$. In addition, $R_{l}, R_{2}$ and $R_{3}$ have nearly zero TCR. $R_{S}, R_{l}$, $R_{2}$ and $R_{3}$, together with the operational amplifier, form a negative feedback loop which requires $R_{S}$ must equal $R_{l}$ when steady state is reached. An important parameter for the operation of shear stress sensor is the overheat ratio (resistive) which is defined as

$$
a_{R}=\left(R_{S}-R_{S 0}\right) / R_{S 0}
$$

where $R_{S}$ is the resistance of the shear stress sensor at the operating temperature and $R s_{0}$ is the resistance at a reference temperature. For example, if we want the overheat ratio to be $10 \%$, then we set $R_{I}=(1+10 \%) R s_{0}$. Due to the negative feedback loop, $V_{\text {out }}$ is tuned automatically to raise the temperature of sensing element so that eventually $R_{S}$ equals $R_{J}$. By measuring $V_{\text {out, }}$ we know how much power is dissipated through fluid and hence know the flow rate.

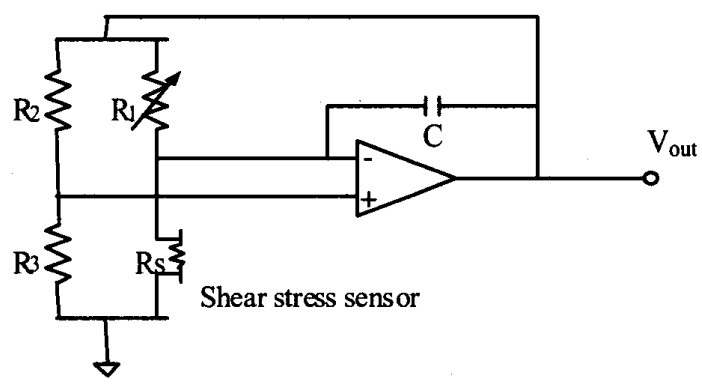

Figure 4 Simplified constant temperature bias circuit.

\section{TESTING AND DISCUSSION}

After calibration of pressure and temperature sensors, we first measured the pressure and temperature distributions as well as the relative output change of the shear stress sensors $\left(\Delta V / V_{0}\right)$ in a fully developed incompressible laminar flow $(Q=1.77$ SLPM). Note $V_{0}$ is the output voltage when the flow rate is zero and $\Delta V$ is the output voltage change at certain flow rate. Figure 5 shows that pressure gradient, temperature and $\Delta V / V_{0}$ are all constant, which agrees well with channel flow theory [6].

Figure 6 shows the pressure difference $\Delta P$ of pressure sensors \#1 and \#5 varying with mass flow rate (in the channel with $h=200 \mu \mathrm{m}$ and $\mathrm{N}_{2}$ flow) both in laminar and turbulent flow regimes. The transition region for laminar and turbulent flow is around $Q=2.5$ SLPM, where $\operatorname{Re}=2100$. The solid line in laminar flow part is the theoretical curve predicted by equation (4). We can 
see that the experimental data agrees with the theory. From laminar to turbulent flow, the slope of the curve increases. Therefore, in the turbulent flow regime, the pressure sensor based flowmeter is more sensitive except that there exists no simple equation for the flow rate.

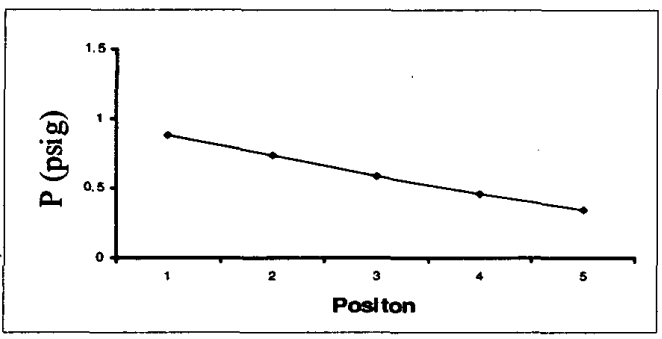

(a) Pressure distribution

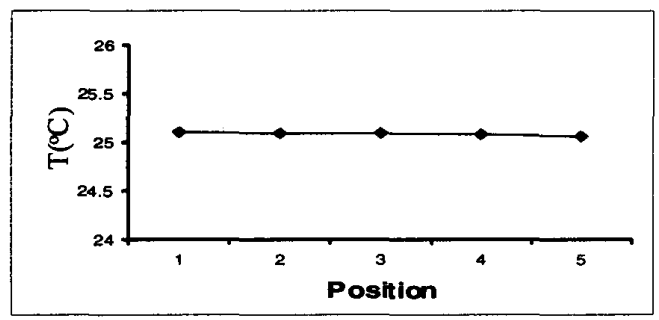

(b) Temperature distribution

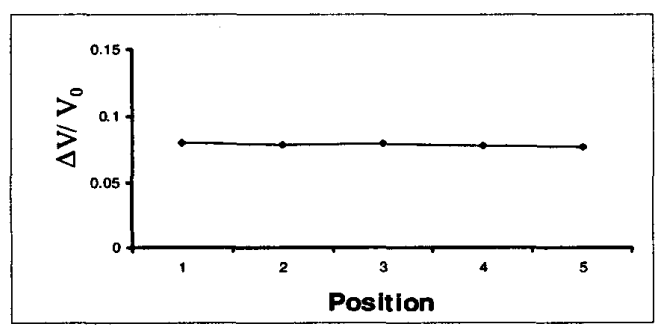

(c) Relative output change of shear stress sensors

Figure 5 Measurement of fully developed incompressible laminar channel flow.

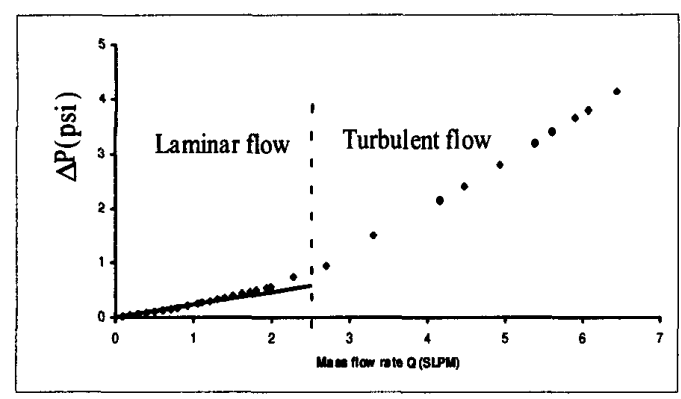

Figure 6 Pressure drop vs. mass flow rate $(h=200$ um and $\mathrm{N}_{2}$ flow).
Figure 7 shows the input power of shear stress sensor measured with $a_{R}=10 \%$ and in a $200 \mu \mathrm{m}$ high channel and nitrogen flow. One of the most severe problems of the hot film shear stress sensors is that the output is very sensitive to ambient temperature. By the on-chip thermistor, the experimental data have been temperature compensated. Interestingly, we find that in the turbulent flow regime, the response of the shear stress sensor is almost linear. The advantage of CT mode is that sensor output will not saturate even for a very large mass flow rate thus the measurement range could be very wide. One thing we should pay attention to is that our thermal shear stress sensor has very short response time, which is an advantage in laminar flow measurement, but in turbulent flow, the sensor output will always fluctuate because of the flow turbulence. Therefore, time averaging is necessary for turbulent flow measurement. Note that in the large flow rate case, the flow becomes both turbulent and compressible and the situation becomes very complicated.

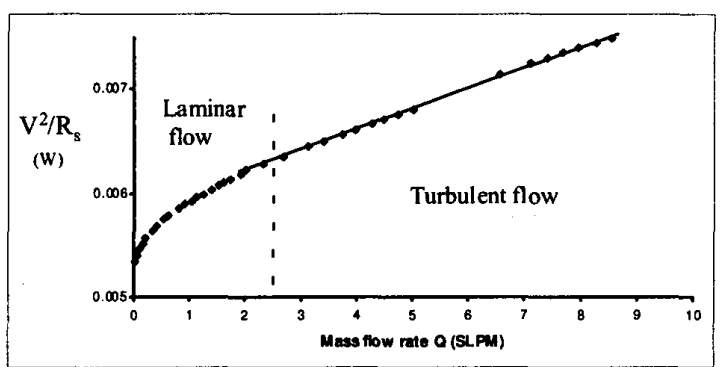

Figure 7 Response of shear stress sensor ( $h=200 \mathrm{um}, a_{R}$ $=10 \%$ and $\mathrm{N}_{2}$ flow).

According to equation (6), $V^{2} / R_{S}$ should be a linear function of $Q^{1 / n}$. Using the least squares method to fit the data, we obtain $n=2.2$ which differs considerably from the conventional value of 3 . This agrees with numerical simulation results, which indicate that $n=$ 2.0. Figure 8 focuses on the laminar flow part of Figure 7 by changing the horizontal axis to $Q^{I / 2.2}$.

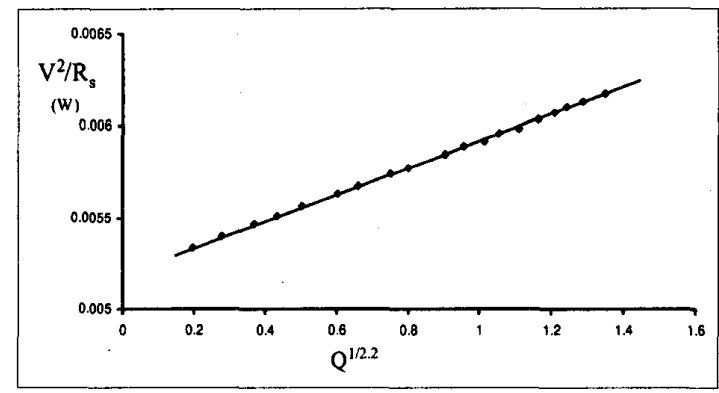

Figure 8 Input power versus $Q^{1 / 2.2}$ (laminar flow region). 
With the vacuum cavity underneath, the heat loss to substrate is reduced significantly so that a very high sensitivity is obtained. It can be seen from Figure 7 that the sensitivity of shear stress sensor is not constant. For example, at $Q=1$ SLPM, the sensitivity is 0.17 V/SLPM but at $Q=0.1$ SLPM, it increases to 0.62 V/SLPM. This mass flowmeter has a higher sensitivity for small flow rate, which is desirable for some applications. Figure 9 shows that the shear stress mass flowmeter has better resolution than that from a pressure sensor in laminar flow regime based on our experimental system.

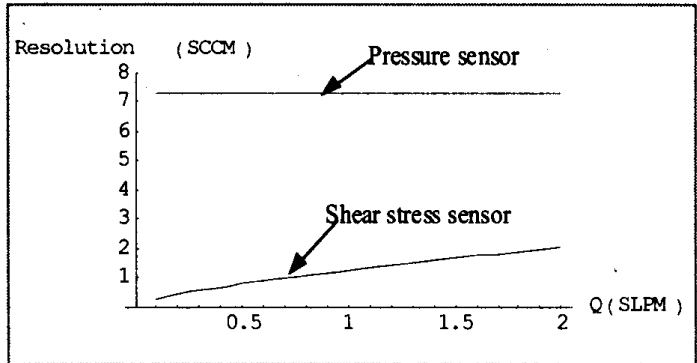

Figure 9 Resolution of shear stress sensor based and pressure sensor based mass flowmeters in laminar flow region.

In order to fully understand the characteristics of the shear stress sensor based flowmeter, extensive tests have been carried out with different overheat ratios, channel heights and gases.

Table 1 compares experimental $A_{0}$ and $B_{0}$ for different overheat ratios both in nitrogen and helium flows in the $200 \mu \mathrm{m}$ high channel. In order to compare $A_{0}$ and $B_{0}$, we need to fix $n$ first. $n$ should not change significantly with overheat ratio or gas, we use the averaged $n$ which is 2.20 .

Table 1 Experimental $A_{0}$ and $B_{0}$ for different $a_{R}(h=200$ $\mu \mathrm{m}$ and $n=2.20$ )

\begin{tabular}{|l|c|c|c|}
\hline gas & $\begin{array}{c}\text { over heat } \\
\text { ratio }\end{array}$ & Ao & Bo \\
\hline Nitrogen & $5 \%$ & 0.349 & 2.84 \\
\cline { 2 - 4 } & $10 \%$ & 0.719 & 5.20 \\
\cline { 2 - 4 } & $20 \%$ & 1.31 & 9.52 \\
\hline \multirow{3}{*}{ Helium } & $5 \%$ & 0.706 & 3.64 \\
\cline { 2 - 4 } & $10 \%$ & 1.31 & 6.87 \\
\cline { 2 - 4 } & $20 \%$ & 2.30 & 13.0 \\
\hline
\end{tabular}

From the above table, we can clearly see that when the overheat ratio doubles, $A_{0}$ and $B_{0}$ approximately double too. This can be explained by following analysis. We know that the resistance of sensing element at temperature $T$ is

$$
R_{s}=R_{S 0}\left[1+\alpha_{T}\left(T-T_{0}\right)\right]
$$

where $R \mathrm{~s}_{0}$ is the resistance at reference temperature $T_{0}$ and $\alpha_{T}$ is the TCR of sensing element. Combined with equation (7), we have

$$
a_{R}=\alpha_{T} \Delta T
$$

According to equation (6), $A_{0}$ and $B_{0}$ are both proportional to $\Delta T$ (note that this is only approximately true because some other terms are also weak functions of temperature) and hence are proportional to $a_{R}$ too.

Table 2 shows experimental $A_{0}$ and $B_{0}$ for different channel heights both in nitrogen and helium flow while the overheat ratio remains $10 \%$. To simplify the comparison, we used the same averaged $n$ as in Table 1. From Equation (6) we have $A_{0} \propto h^{-2 / n}$. In Table 2 we only see that $A_{0}$ increases as $h$ decreases however it is difficult to tell how it changes with $h$ from our data. One possible explanation is the change of $h$ will affect the heat transfer in the channel and accordingly cause other parameters to change.

Table 2 Experimental $A_{0}$ and $B_{0}$ for different channel heights $\left(a_{R}=10 \%\right.$ and $\left.n=2.20\right)$

\begin{tabular}{|l|c|c|c|}
\hline gas & $\begin{array}{c}\text { channel } \\
\text { height }\end{array}$ & Ao & Bo \\
\hline Nitrogen & $150 \mathrm{um}$ & 0.789 & 5.61 \\
\cline { 2 - 4 } & $200 \mathrm{um}$ & 0.719 & 5.20 \\
\cline { 2 - 4 } & $288 \mathrm{um}$ & 0.562 & 5.48 \\
\hline \multirow{3}{*}{ Helium } & $150 \mathrm{um}$ & 1.51 & 7.25 \\
\cline { 2 - 4 } & $200 \mathrm{um}$ & 1.31 & 6.87 \\
\cline { 2 - 4 } & $288 \mathrm{um}$ & 0.847 & 7.38 \\
\hline
\end{tabular}

Different gas properties will also affect the sensor sensitivity. It can be shown that $A_{0} \propto k_{T} \alpha^{-1 / n}$, where $\alpha=k_{T} / \rho c_{P}$ is the thermal diffusivity of the gas. For nitrogen, $k_{T}=25.9 \times 10^{-3} \mathrm{~W} / \mathrm{m} \cdot \mathrm{K}, \alpha=22.1 \times 10^{-6}$ $\mathrm{m}^{2} / \mathrm{s}$ and for helium, $k_{T}=152 \times 10^{-3} \mathrm{~W} / \mathrm{m} \cdot \mathrm{K}, \alpha=$ $180 \times 10^{-6} \mathrm{~m}^{2} / \mathrm{s}$ and let $n=2.20$, we obtain the theoretical ratio of $A_{0}(\mathrm{He})$ over $A_{0}\left(\mathrm{~N}_{2}\right)$ is 2.26 . From both Table 1 and Table 2, we can see that all the experimental ratios are less than the theoretical value, which also implies that more parameter changes should be considered. 


\section{CONCLUSION}

A novel mass flowmeter using a multi-sensor chip has been successfully fabricated. This shear stress sensor based mass flowmeter is not intrusive thus the disturbance to flow is minimized. With the vacuum cavity underneath, the heat loss to substrate is significantly reduced and the sensitivity is increased considerably. In addition, the output of shear stress sensors has been temperature compensated by the onchip thermistor. Our study also shows that mass flowmeter using shear-stress sensors produces a much better resolution than that from pressure sensors in laminar flow regime. Extensive tests have been carried out with various overheat ratios, channel heights and gases. Since the behavior of the pressure sensor is well established, it can be used for on-device calibration as well as pressure compensation. In this way, the performance of this mass flowmeter could be further improved.

\section{ACKNOWLEDGEMENTS}

This work is supported by JPL under account code 49 . 204-52000-0-3460. The authors would like to thank Shuyun Wu, Trevor Roper and Hung Bui for their help with the process, Xing Yang and Ellis Meng for their help with the paper.

\section{REFERENCE}

[1] R. J. Goldstein, Fluid Mechanics Measurements, $2^{\text {nd }}$ Edition, Taylor \& Francis, 1996

[2] T. S. J. Lammerink, N. R. Tas, M. Elwenspoek and J. H. J. Fluitman, Mico-liquid flow sensor, Sensors and Actuators A, 37-38 (1993) 45-50

[3] N. T. Nguyen, Micormachined flow sensors - a review, Flow Meas. Instrum. Vol. 8, No. 1. pp. 716,1997

[4] F. Jiang, Silicon-Micromachined Flow Sensors, Ph.D. Thesis, California Institute of Technology, 1998.

[5] J. Q. Liu, Integrated Micro Devices for Small Scale Gaseous Flow Study, Ph.D. Thesis, California Institute of Technology, 1995.

[6] F. M. White, Fluid mechanics, $2^{\text {nd }}$ Edition, McGraw-Hill, 1986. 